Original Research

\title{
Short-Term Straw Returning Improves Quality and Bacteria Community of Black Soil in Northeast China
}

\author{
Liu Yaliang', Gu Yan ${ }^{1 *}$, Wu Chunsheng', Zhao Hongxiang², Hu Wenhe', \\ Xu Chen ${ }^{1,2}$, Chen Xifeng ${ }^{1}$ \\ ${ }^{1}$ Agricultural College, Jilin Agricultural University, Xin Cheng Street No. 2888, Changchun, Jilin, 130012, China \\ ${ }^{2}$ Jilin Academy of Agricultural Science, Shengtai Street No. 1363, Changchun, Jilin, 130033, China
}

Received: 15 May 2021

Accepted: 21 September 2021

\begin{abstract}
Despite our extensive knowledge on the effects of long-term straw returning on soil, little is known about the effects of short-term straw returning. We identified three study sites and evaluated their soil organic matter $(\mathrm{OM}), \mathrm{pH}$, and enzyme activity after two years of straw returning treatment. Bacterial diversity and community were determined by 16s RNA sequencing. We observed elevated OM content and $\mathrm{pH}$ after short-term straw returning treatment. The bacterial phyla Proteobacteria and Bacteroidetes exhibited similar dynamics on straw application. We observed reduced levels of Actinobacteria and Chloroflexi bacterial phyla after straw returning. Reduced Actinobacteria and Chloroflexi may be due to competition from dominant bacteria. In general, OM content and enzyme activity had the same trend that closely correlated with the amount and community of microorganisms in the soil. Proteobacteria and Bacteroidetes were the critical phyla in straw degradation and might improve soil OM content. Proteobacteria and Actinobacteria were identified as copiotrophic taxa. In summary, straw returning treatment might maintain soil stability and bacterial diversity better. The bacterial phyla Proteobacteria and Bacteroidetes were dominant over other microbial fractions during straw decomposition. The survival competition might be one of the main reasons for the decrease of Actinobacteria and Chloroflexi. Short-term straw returning to the field can markedly improve soil quality. However, soils in different locations respond differently to straw return practices and their responses are influenced by the soil's basic parameters and climate.
\end{abstract}

Keywords: crop straw returning, soil bacteria diversity, bacteria community, soil organic matter

*e-mail: guyan810831@163.com 


\section{Introduction}

Dubbed the "giant panda of cultivated land", the black soil in Northeast China is one of four major chernozem regions around the world. Covering an area of 1.09 million square kilometers, it is also one of the most fertile regions in the country. Black soil is recognized as the most fertile soil in the world. It takes 200 to 400 years to form a 1-centimeter-thick layer of black soil under natural conditions, according to a white paper released by the Chinese Academy of Sciences last month [1]. However, irrational cultivation and tillage, as well as climate change, have led to the degradation of black soil in Northeast China [2]. And in recent years, this situation has become more and more serious. In order to protect the black soil in Northeast China, a variety of measures have been actively applied to reduce the loss of black soil, among which straw returning to the field is one of the reliable ways.

Crop straw returning to the field may effectively improve soil fertility, soil carbon sequestration and sustain soil productivity $[3,4]$. Once returned to the field, straw gradually decomposes into organic matter $(\mathrm{OM})$, which greatly influences soil function and quality. Straw returning is reported to significantly increase soil OM content relative to the application of chemical fertilizers only [5]. High soil OM content can increase soil nutrients supply [6,7], improve soil physical and biological properties [8], and enhance soil buffering capacity [9]. There is a close correlation between soil microorganisms and soil OM. Soil microorganisms are the main drivers of carbon dynamics and nutrient turnover during biogeochemical cycling $[10,11]$. As the main decomposers in soil, bacteria are dominant players in the initial phases of straw decomposition, although some fungi, which decompose more recalcitrant materials dominate later stages [12-15]. Additionally, soil microorganisms are important in soil aggregation and soil structure formation [16]. Thus, microorganisms are regarded as architects of surrounding soil environments [17]. A wide body of evidence shows that soil microorganisms are crucial for soil fertility. Straw degradation products can influence soil microorganisms by modulating stability and diversity of soil microbial structure [18]. This can promote the development of an excellent virtuous circle of soil microorganism-soil OM-straw degradation. Northeast China is an extensive region that generates a lot of crop straw every year. Straw returning may not only save manpower, but also improve soil OM. While multiple studies have shown the long-term effects of straw returning on soil, few have examined the effects of short-term straw returning.

In this study, straw was returned to fields where it had not been previously returned. The study was carried out at 3 sites in Northeast China, located in Songyuan, Dunhua and Jiutai, which are typical black soil regions. We hypothesized that short term straw returning and deep ploughing may improve soil quality. However, whether responses from the different regions are consistent merits further study. Importantly, using highthroughput sequencing technology, we comprehensively examined the effects of short-term straw returning on soil bacteria communities. Our data offer novel insights on short-term straw returning practices.

\section{Experimental}

\section{Description of Study Sites and Soil Sampling Strategy}

This study was done at 3 locations in Jilin Province, China: Songyuan city $\left(45^{\circ} 17^{\prime} \mathrm{N}, 124^{\circ} 80^{\prime} \mathrm{E}\right.$, marked as A), Dunhua city $\left(43^{\circ} 25^{\prime} \mathrm{N}, 128^{\circ} 21^{\prime} \mathrm{E}\right.$, marked as B), and Jiutai city $\left(44^{\circ} 8^{\prime} \mathrm{N}, 126^{\circ} 49^{\prime} \mathrm{E}\right.$, marked as C). Although these locations belong to different ecological areas, their

a)

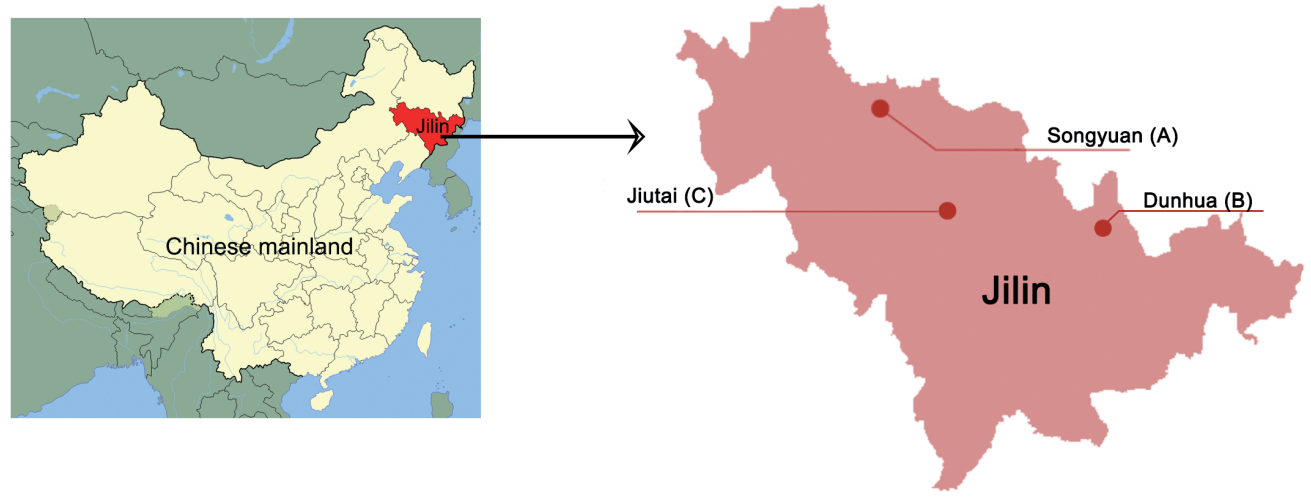

b)

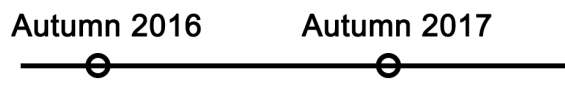

Jun 2018

Oct 2018

Straw Return in Deep Straw Return in Deep

Sampling 6 times

Fig. 1. Location and design. A shows the sampling location, B shows the straw returning treatment and sampling time. 
soil types are typical Northeast China black soil. The 3 locations have been treated with inorganic fertilizer for $>20$ years and straw had not been previously returned to the fields. The study used a randomized complete block design. With or without straw returning were considered random effects. There were 6 blocks at each location, 3 controls and 3 experiment groups. A two years short-term deep tillage with straw returning was done in autumn of 2016 and 2017 (Fig. 1). Maize straw was crushed to $5 \mathrm{~cm}$ sizes after harvest and evenly sprinkled in the field. In spring of the next year, the straw was ploughed into the soil using a fence hydraulic turning plow (1LYFT-450, Longfeng, China) at a ploughing depth of $35-40 \mathrm{~cm}$. Soil samples from fields in Songyuan, Dunhua and Jiutai that underwent short-term straw returning were marked A, B, and C, respectively. Soil samples from fields in Songyuan, Dunhua and Jiutai without short-term straw returning were marked A-CK, B-CK, and C-CK, respectively. All soil samples were collected from June 2018 to October 2018. Sampling was done every 25 days, 6 times in total (Jun. 26, jointing stage; Jul. 21, trumpet stage; Aug. 15, spinning stage; Sep. 9, grouting period; Oct. 4, milk-ripening stage; Oct. 29, fullripening stage). At each sampling time, 30 samples from each treatment, at each location were collected using the chessboard method and 3 samples randomly selected for $16 \mathrm{~S}$ sequencing. In total, 108 samples were collected [36 samples for A, B and C, 6 samples (3 treatment and $3 \mathrm{CK}$ ) per time per location, 6 times in total]. The other samples were used to analyze soil enzyme activity and organic matter content. All samples were stored at $-80^{\circ} \mathrm{C}$ until use.

\section{Soil Properties and Soil Enzymes Activity Analysis}

Soil enzyme activity is an important index of its biological activity and fertility. To examine this important indicator, we analyzed the soil for the enzymatic activities of invertase, urease, and alkaline phosphatase as described by Yang [19] and Geisseler [20]. Soil organic matter $(\mathrm{OM})$ content is a major parameter in soils and agriculture in general. OM content under various fertilization strategies was determined using the potassium dichromate method as described before [21]. Total nitrogen (TN) was determined by Kjeldahl method; Total phosphorus (TP) was determined using sulfuric acid-perchloric acid digestion method; Total potassium (TK) was determined using the sodium bicarbonate extraction-molybdenum-antimony antispectrophotometric method; Additionally, soil water content (SWC, \%) at each location and sampling time were measured using the oven-drying method. First, wet soil (W1) was weighed in an aluminum box using an electronic balance and the soil dried for $12 \mathrm{~h}$ at $105^{\circ} \mathrm{C}$, until constant weight was reached. Next, the dry soil was weighed in the aluminum box (W2) using an electronic balance. Finally, the aluminum box (W3) was weighed using electronic balance. SWC was calculated using the equation: $\mathrm{SWC} \%=(\mathrm{W} 1-\mathrm{W} 2) /(\mathrm{W} 2-\mathrm{W} 3)$. Soil $\mathrm{pH}$ was determined using a $\mathrm{pH}$ tester (Takemura Electric Works Ltd.).

\section{Isolation of Total Microbial DNA}

Soil microbial genomic DNA was extracted using a MOBIO PowerSoil DNA Isolation Kit (Qiagen) following manufacturer instructions. Agarose gel electrophoresis was used to assess DNA integrity. DNA was quantified using Qubit2.0 DNA Assay Kit (Sangon Biotech Co., Ltd). The primers 341f (CCTACGGGNGGCWGCAG) and $805 \mathrm{R}$ (GACTACHVGGGTATCTAATCC) were used to amplify the V3-V4 region of the 16S rRNA gene [22]. PCR amplification was done using an Eppendorf mastercycler in a $50 \mu \mathrm{L}$ reaction volume comprised of 10ng genomic DNA, $0.5 \mu \mathrm{L}$ dNTP (10mM each), $0.5 \mu \mathrm{L}$ of each PCR primer $(50 \mu \mathrm{M})$, and $0.5 \mu \mathrm{L}$ Taq $(5 \mathrm{U} / \mu \mathrm{L})$. Cycling conditions were as follows: initial denaturation for $10 \mathrm{~min}$ at $95^{\circ} \mathrm{C}$, followed by 30 cycles at $95^{\circ} \mathrm{C}$ for $15 \mathrm{~s}$, annealing at $60^{\circ} \mathrm{C}$ for $15 \mathrm{~s}$ and extension at $72^{\circ} \mathrm{C}$ for $30 \mathrm{~s}$ and final extension at $72^{\circ} \mathrm{C}$ for $5 \mathrm{~min}$. A gel extraction kit (Axygen) was used to recover desired DNA fragments after electrophoresis. DNA concentration after gel extraction was determined using Qubit2.0 (Life Tech) and DNA quality determined using an Agilent 2100 Bioanalyzer (Agilent). Quantitative PCR (qPCR) was used to test the efficiency of adapters. Based on the efficiency, clone libraries were diluted to a concentration of $1 \mathrm{ng} / \mu \mathrm{l}$ for sequencing. Hiseq 2500 (Illumina) was used for pair-end (PE) 250bp sequencing.

\section{Sequence Data Analysis}

Sequencing data was separated by barcode annotation and PCR primer sequences, which were then depleted. Data splicing and quality filtering were done using the FLASH (v1.2.7), Qiime (v1.9.1) and UCHIME algorithms (v4.1), respectively. Operational taxonomic units (OTUs) clustering was done using Uparse (version 7.0.1001; http://drive5.com/uparse/) based on $97 \%$ identity threshold. OTU abundance (reads number) in each sample was calculated and OTUs with $>2$ reads used for further analysis. Next, alpha diversity indicators (Chaol, ACE, observed OTUs, Shannon and Simpson) of the sequencing data within each group $(\mathrm{n}=3)$ and beta diversity index (Unweighted UniFrac distance) for each sample were calculated. Principal Co-ordinates Analysis (PCoA) of the samples was done using Unweighted UniFrac distance of beta diversity index. SILVA rRNA database (http://www.arb-silva. de/) on Mothur website (http://www.mothur.org/wiki/ RDP_reference_files) was queried for OTU annotations. OTUs relative abundances (phylum $\sim$ species level) was calculated and taxonomy assignment (phylum $\sim$ species level) done using the Ribosomal Database Project (RDP) classifier ( $80 \%$ confidence). A linear model with redundancy analysis (RDA) was used to assess 


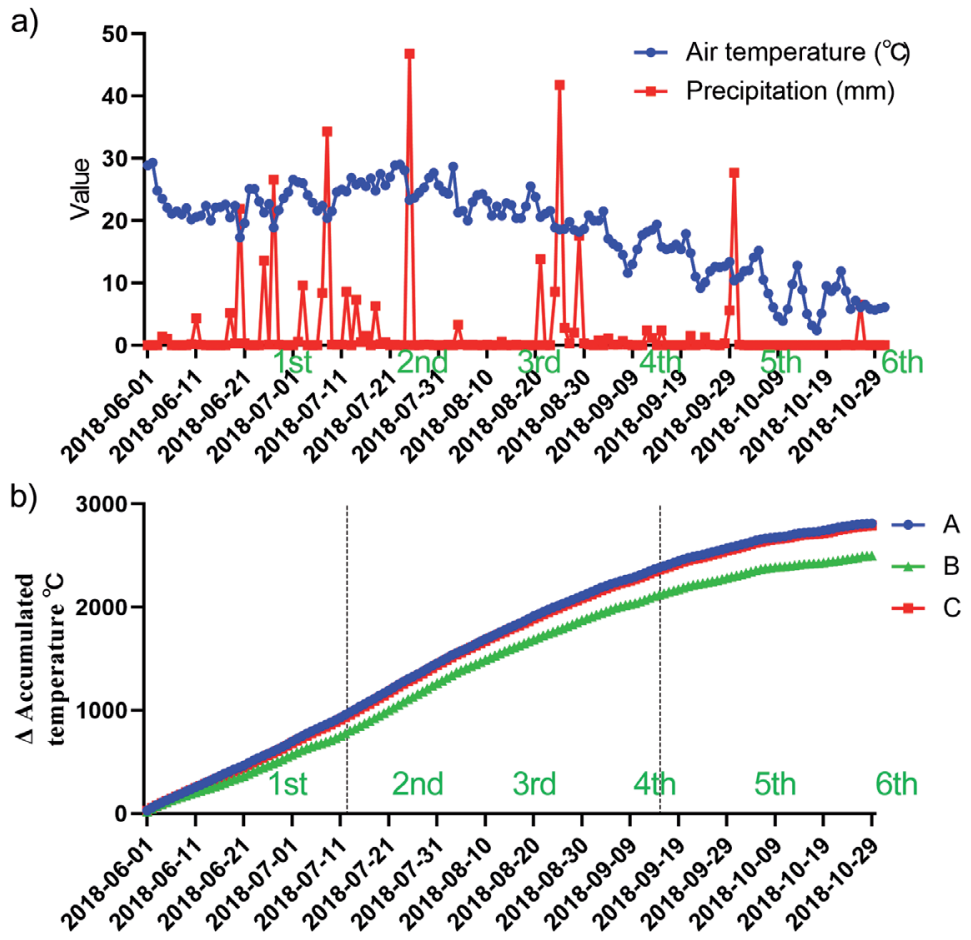

Fig. 2. Temperature and precipitation. a) show the air temperature and precipitation in different months. b) show the accumulated temperature from June 1 to September 30. The accumulated temperature before June 1st defaults to 0 . The green arrow indicates the sampling time.

the relationships between environmental factors and species abundance as previously described [23].

\section{Results}

\section{Soil Characteristics After Short-Term Straw Returning Treatment}

The temperature and precipitation analysis during the experiment revealed gradual temperature increase from June to July, and gradually decrease from August to October. Precipitation was highest in June-August. Temperature gradually plateaued in late September (Fig. 2 a-b).

The short-term straw returning treatment significantly affected soil OM content. As expected, OM content increased after short-term straw returning at all experiment locations and sampling times (Fig. 3 a-c), with $\mathrm{OM}$ content peaking at $3^{\text {rd }}$ sampling at $\mathrm{A}, \mathrm{B}$, and C. The difference between the control and the treatment group was highest at $3^{\text {rd }}$ sampling at locations $\mathrm{A}$ and $\mathrm{C}$, and at $4^{\text {th }}$ sampling at location B (Fig. 3 a-c). Analysis of $\mathrm{pH}$ revealed that soil was weakly alkaline at location A and weakly acidic at location B and C. However, soil $\mathrm{pH}$ at the 3 locations declined at varying degrees after short-term straw returning (Fig. 3 d-f). Additionally, short-term straw returning significantly increased SWC at location $\mathrm{A}, \mathrm{B}$ and $\mathrm{C}$ at $2^{\text {nd }}-6^{\text {th }}$ sampling, indicating the benefit of short-term straw returning (Fig. 3 g-i).
Soil enzyme activity is an important index in the evaluation of soil fertility. Our results show that straw returning enhanced the activity of these four soil enzymes (S-AI, S-AKP, CAT and UE) to varying degrees at study locations (Fig. 4 a-c). Additionally, enzyme activity peaked at the $3^{\text {rd }}$ or $4^{\text {th }}$ sampling time.

\section{Sequence Data Summary}

A total of 11,959,033 sequence reads were obtained from the 108 soil samples. After trimming sequence adapters and filtering out low-quality reads, 11,667,501 tags (an average of 108,032 tags per sample) remained (Table S1). Rarefaction curves (Fig. S1a) and species accumulation curves (Fig. S1b) showed that increased OTUs tended to be flat, with increasing sequencing read number and soil samples, respectively. Indicating that the amount of data and sequenced samples were sufficient. Additionally, the PCA and phylogenetic analysis results showed that all the samples in the same group were clustered together, revealing the excellent repeatability in this study (Fig. S2). All the basic statistical results indicated the excellent sequence data quality and consistency of the repetitions.

\section{Effects of Short-Term Straw Returning on Bacterial Diversity}

Short-term straw returning significantly influenced soil bacteria diversity, but different regions showed 
OM

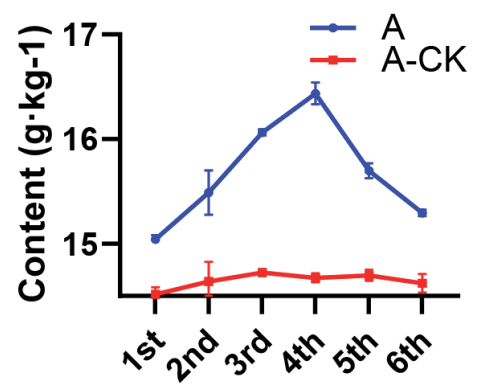

Sampling time

pH

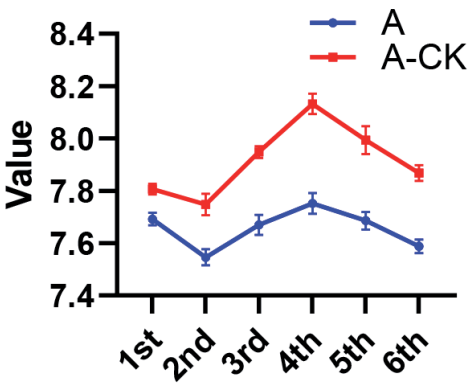

Sampling time

SWC

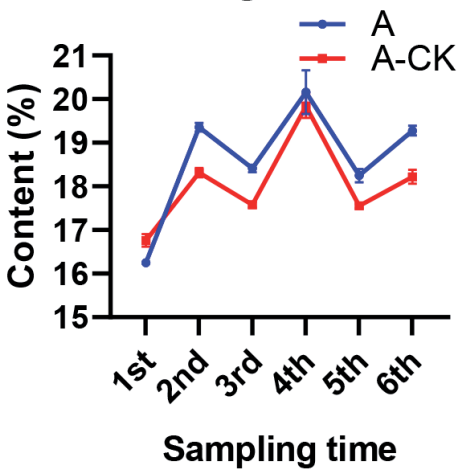

OM

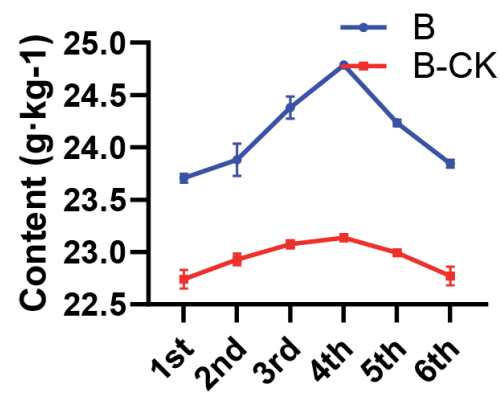

Sampling time

$\mathrm{pH}$

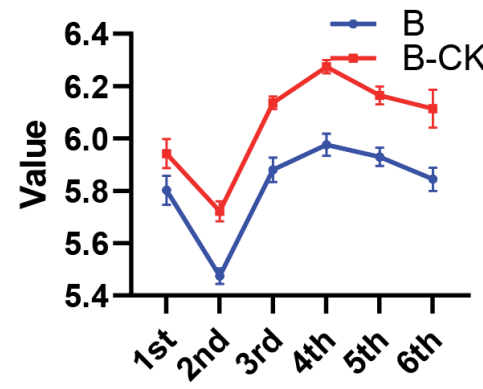

Sampling time

SWC

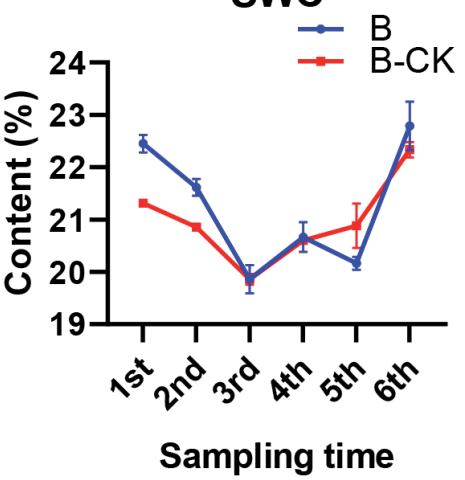

OM

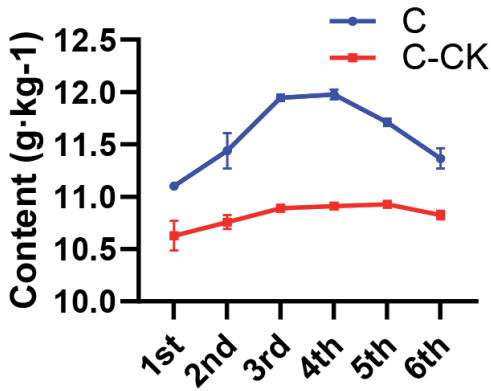

Sampling time

pH

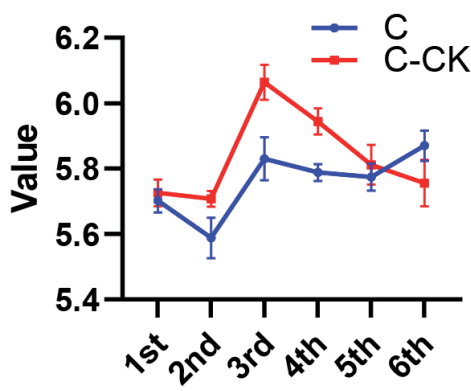

Sampling time

SWC

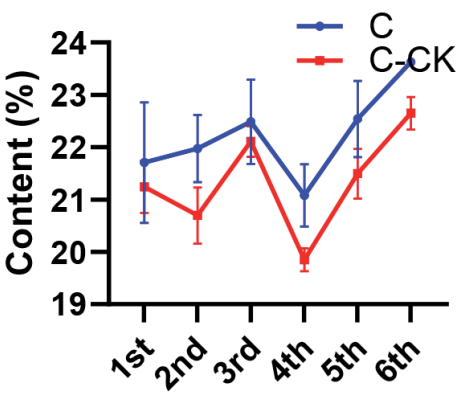

Sampling time

Fig. 3. Soil physical and chemical properties. OM: organic matter, SWC: soil water content. a, b, and c denotes soil samples from Songyuan, Dunhua, and Jiutai, respectively. CK denotes soil samples without straw return.

different patterns. At location A, short-term straw returning significantly reduced the $\alpha$-diversity indexes, including chaol, observed species, and shannon, especially at the $1^{\text {st }}, 2^{\text {nd }}, 3^{\text {rd }}$ and $5^{\text {th }}$ sampling (Table 1 ). At location $\mathrm{B}$ and $\mathrm{C}$, the bacteria diversity indexes significantly increased upon short-term straw returning. Additionally, there were great differences between different sampling times. $\alpha$-diversity indexes, including chaol, goods coverage, observed species, and shannon, at $1^{\text {st }}, 2^{\text {nd }}, 3^{\text {rd }}, 4^{\text {th }}$ and $5^{\text {th }}$ sampling were significantly higher than at $6^{\text {th }}$ sampling in group A, A-CK, B, B-CK, and $\mathrm{C}-\mathrm{CK}$ (both in control and treatment groups). In group $\mathrm{C}$ (treated with short-term straw returning), no significant difference was observed across sampling times (Table 1).
Effects of Short-Term Straw Returning on Bacterial Communities

16s RNA sequencing revealed that bacterial composition changed after short-term straw returning, with some bacteria exhibiting similar changing patterns at the 3 locations. Our data show that Proteobacteria, Actinobacteria, Acidobacteria, Bacteroidetes, Gemmatimonadetes and Chloroflexi were the dominant bacteria phyla (Fig. 5 a-c).

Short-term straw returning significantly increased Proteobacteria, except at the $1^{\text {st }}$ sampling time at location B. Similarly, Bacteroidetes and Gemmatimonadetes were significantly increased upon short-term straw returning. However, relative abundance 
a)

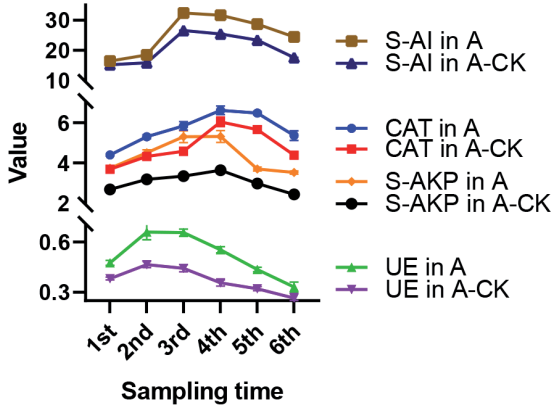

b) Location B

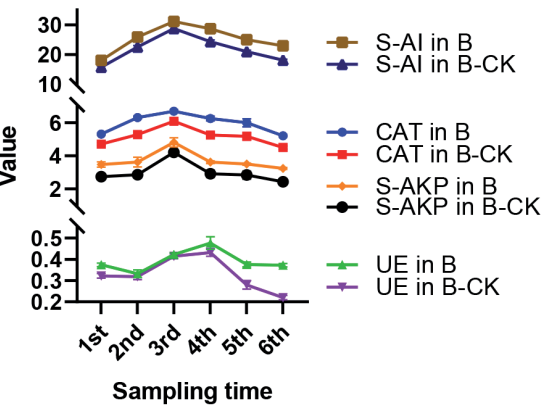

C) Location C

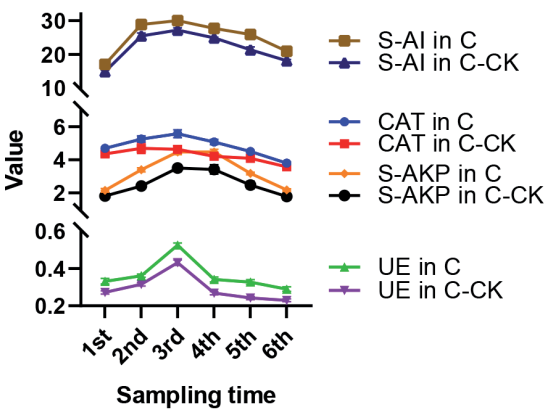

Fig. 4. Soil enzyme activity. S-AI: solid-acid invertase $\left(\mathrm{mg}\right.$ glucose $\left.\mathrm{g}^{-1} \cdot 24 \mathrm{~h}^{-1}\right)$, S-AKP: soil-alkaline phosphatase $\left(\mathrm{mg}\right.$ phenol g $\left.\mathrm{g}^{-1} \cdot 2 \mathrm{~h}^{-1}\right)$, CAT: catalase $\left(0.1 \mathrm{~mol} \mathrm{KMnO}_{4} \mathrm{~g}^{-1}\right.$ soil. $\left.30 \mathrm{mim}^{-1}\right)$, UE: urease $\left(\mathrm{mg} \mathrm{NH}_{3}-\mathrm{N} \mathrm{g}^{-1} .3 \mathrm{~h}^{-1}\right)$. a, b, and c notes soil samples from Songyuan, Dunhua and Jiutai, respectively. CK denotes soil samples without straw return.

of Actinobacteria and Chloroflexi was decreased, and was most obvious at locations A and C. Additionally, we found that the relative abundance of the dominant bacteria varied widely across sampling times in the control, while upon short-term straw returning, relative abundance was stable at various sampling times, indicating the stability of the bacteria communities. Additionally, we calculated the significance of relative abundance of dominant bacteria in different groups (Fig. 6 a-c). For bacteria with low relative abundance, the effect of short-term straw returning treatment was also great. While we did not identify most bacteria at

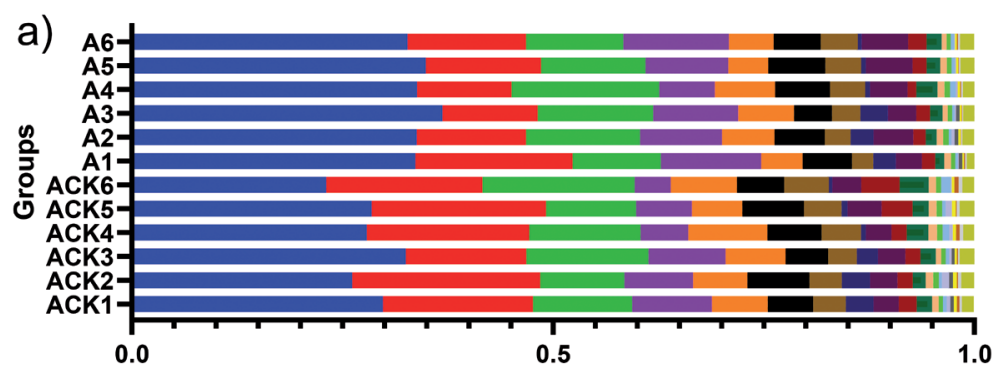

- Others

- p_Tectomicrobia

- P_GAL15

(1) $\mathrm{p}$-Elusimicrobia

- $\mathrm{p}$ - Tenericutes

- P_Euryarchaeota

- P__Armatimonadetes

- $p$ unidentified

- p Nitrospirae

- $\mathrm{p}$ __Latescibacteria
- p Thaumarchaeota

- p_-Verrucomicrobia

- p_Eirmicutes

- p_Planctomycetes

- p Chloroflexi

- p___Gemmatimonadetes

- $\mathrm{p}$ __Bacteroidetes

- $p$ Acidobacteria

p Actinobacteria

- $p$ __Proteobacteria

Relative abundance (\%)

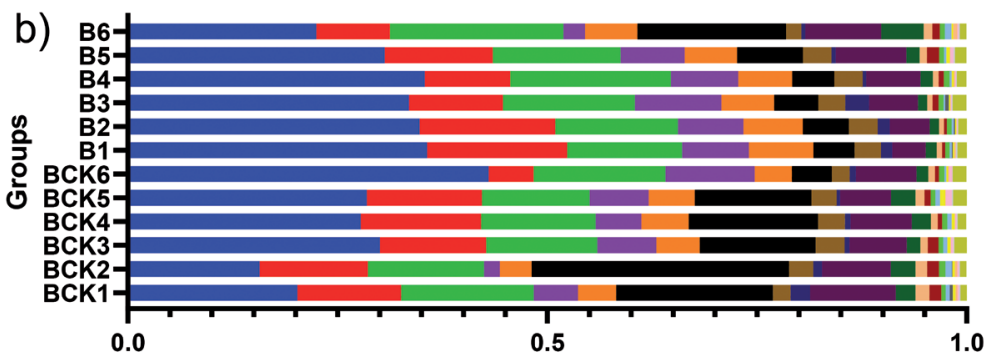

Others

p__Tectomicrobia

p-Parcubacteria

p - GAL15

1 $\mathrm{p}$ __Elusimicrobia

p_-Tenericutes

p Latescibacteria

- p_Armatimonadetes

- p_-Thaumarchaeota

- $p$ unidentified
- p_Planctomycetes

- p_Firmicutes

- $p$ - Nitrospirae

- p_-Gemmatimonadetes

- $\mathrm{p}$ __Bacteroidetes

- $p$ - Verrucomicrobia

- p Actinobacteria

- p_Acidobacteria

- $p$ __Proteobacteria

- $p$-Chloroflexi

Relative abundance (\%)

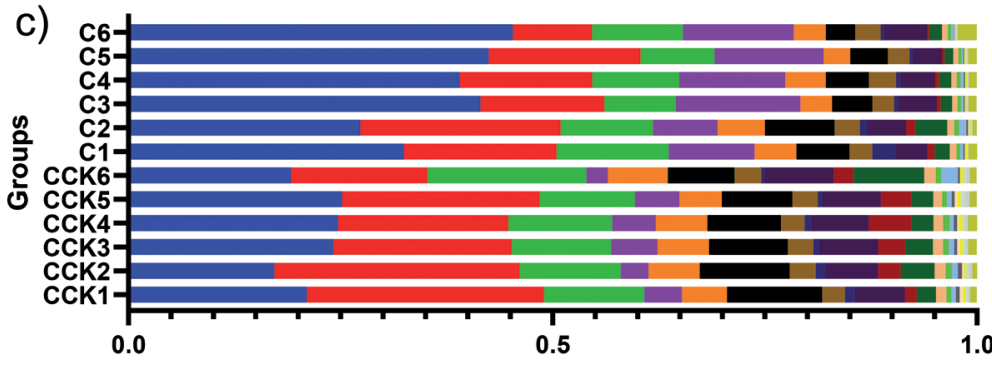

- Others

p_Parcubacteria

p__Omnitrophica

$\mathrm{p}$ _Elusimicrobia

p Euryarchaeota

- p-Tectomicrobia

- p Latescibacteria

- p-Armatimonadetes

- p-Firmicutes

- $p_{\text {__unidentified }}$
- p Thaumarchaeota

- $p$ _ Nitrospirae

- p_Planctomycetes

- $p$ - Bacteroidetes

- $p$ Gemmatimonadetes

- $\mathrm{p}$ __Verrucomicrobia

- p_Chloroflexi

$\mathrm{p}$-Acidobacteria

- p Actinobacteria

- P__ Proteobacteria

Relative abundance (\%)

Fig. 5. Effects of short-term straw returning on bacterial communities. a), b), and c) notes soil samples at Songyuan, Dunhua, and Jiutai, respectively. CK denotes soil samples without straw return. 
Table 1. Effects of short-term straw returning on bacterial diversity.

\begin{tabular}{|c|c|c|c|c|c|c|c|}
\hline & & & chao1 & Goods_coverage & Observed_species & PD_whole_tree & Shannon \\
\hline \multirow{18}{*}{ Control } & \multirow{6}{*}{ A } & $1^{\text {st }}$ & $8093.70 \pm 146.35 \mathrm{ab}^{* *}$ & $0.9165 \pm 0.0018 \mathrm{ab}$ & $5013.13 \pm 178.26 \mathrm{ab}^{*}$ & $303.72 \pm 12.39 \mathrm{ab}$ & $10.7566 \pm 0.1024 \mathrm{ab}^{*}$ \\
\hline & & $2^{\text {nd }}$ & $8323.57 \pm 83.07 \mathrm{a}^{* *}$ & $0.9138 \pm 0.0009 \mathrm{a}$ & $5168.57 \pm 60.31 \mathrm{a}^{* *}$ & $310.77 \pm 5.57 \mathrm{a}$ & $10.8622 \pm 0.0486 \mathrm{a}$ \\
\hline & & $3^{\text {rd }}$ & $7765.88 \pm 340.56 \mathrm{ab}^{*}$ & $0.9201 \pm 0.0036 \mathrm{ab}$ & $4817.53 \pm 205.01 \mathrm{ab}^{*}$ & $285.33 \pm 9.26 \mathrm{cbd}$ & $10.6907 \pm 0.1149 \mathrm{ab}$ \\
\hline & & $4^{\text {th }}$ & $7739.30 \pm 598.15 \mathrm{ab}$ & $0.9203 \pm 0.0060 \mathrm{ab}$ & $4797.40 \pm 310.99 \mathrm{ab}$ & $282.70 \pm 12.03 \mathrm{cb}$ & $10.6209 \pm 0.2275 \mathrm{ab}$ \\
\hline & & $5^{\text {th }}$ & $7939.45 \pm 322.88 \mathrm{ab}^{* *}$ & $0.9180 \pm 0.0025 \mathrm{ab}$ & $5004.37 \pm 108.32 \mathrm{a}^{* *}$ & $293.60 \pm 7.29 \mathrm{ab}^{*}$ & $10.7865 \pm 0.0669 \mathrm{a}^{*}$ \\
\hline & & $6^{\text {th }}$ & $7072.08 \pm 564.65 \mathrm{~b}$ & $0.9278 \pm 0.0057 \mathrm{~b}$ & $4405.70 \pm 274.49 b$ & $267.31 \pm 6.42 \mathrm{~cd}$ & $10.3610 \pm 0.1964 \mathrm{~b}$ \\
\hline & \multirow{6}{*}{ B } & $1^{\text {st }}$ & $6111.28 \pm 193.27 \mathrm{a}$ & $0.9428 \pm 0.0020 \mathrm{ab}$ & $3913.03 \pm 188.55 \mathrm{a}$ & $222.32 \pm 8.42 \mathrm{ab}$ & $9.8538 \pm 0.2324 \mathrm{ab}$ \\
\hline & & $2^{\text {nd }}$ & $3536.99 \pm 1240.52 \mathrm{~b}$ & $0.9695 \pm 0.0149 \mathrm{bc}$ & $2443.43 \pm 586.56 \mathrm{~b}$ & $163.12 \pm 28.25 \mathrm{c}$ & $8.7413 \pm 0.3136 \mathrm{~d}$ \\
\hline & & $3^{\text {rd }}$ & $6436.52 \pm 144.76 \mathrm{a}$ & $0.9396 \pm 0.0014 \mathrm{a}$ & $4217.07 \pm 196.20 \mathrm{a}$ & $228.33 \pm 9.15 \mathrm{ab}$ & $10.1842 \pm 0.2812 \mathrm{ab}$ \\
\hline & & $4^{\text {th }}$ & $6384.03 \pm 124.69 \mathrm{a}$ & $0.9401 \pm 0.0015 \mathrm{a}$ & $4189.13 \pm 17.42 \mathrm{a}$ & $222.32 \pm 2.64 \mathrm{~b}$ & $10.1199 \pm 0.0417 \mathrm{~b}$ \\
\hline & & $5^{\text {th }}$ & $6283.97 \pm 252.38 \mathrm{a}$ & $0.9412 \pm 0.0027 \mathrm{ab}$ & $4221.80 \pm 168.89 \mathrm{a}$ & $237.19 \pm 6.53 \mathrm{a}$ & $10.1907 \pm 0.1179 \mathrm{~b}$ \\
\hline & & $6^{\text {th }}$ & $4878.61 \pm 85.16 \mathrm{~b}$ & $0.9559 \pm 0.0008 \mathrm{c}$ & $3263.90 \pm 50.73 \mathrm{~b}$ & $193.65 \pm 3.81 \mathrm{c}$ & $9.6650 \pm 0.1614 \mathrm{c}$ \\
\hline & \multirow{6}{*}{$\mathrm{C}$} & $1^{\text {st }}$ & $6081.14 \pm 127.20 \mathrm{a}$ & $0.9298 \pm 0.0018 \mathrm{ab}$ & $3773.93 \pm 117.04 \mathrm{ac}$ & $224.61 \pm 6.96 \mathrm{ab}$ & $10.0965 \pm 0.1135 \mathrm{ab}$ \\
\hline & & $2^{\text {nd }}$ & $5920.38 \pm 255.72 \mathrm{ab}$ & $0.9318 \pm 0.0035 \mathrm{ab}$ & $3694.10 \pm 217.40 \mathrm{abc}$ & $221.50 \pm 11.91 \mathrm{ab}$ & $9.9567 \pm 0.1758 \mathrm{ab}$ \\
\hline & & $3^{\text {rd }}$ & $6175.58 \pm 205.54 \mathrm{a}$ & $0.9289 \pm 0.0020 \mathrm{a}$ & $3825.67 \pm 92.81 \mathrm{ac}$ & $222.67 \pm 3.81 \mathrm{ab}$ & $10.0881 \pm 0.1443 \mathrm{ab}$ \\
\hline & & $4^{\text {th }}$ & $6377.73 \pm 91.33 \mathrm{a}$ & $0.9259 \pm 0.0016 \mathrm{a}$ & $4026.47 \pm 69.46 \mathrm{ab}$ & $229.83 \pm 4.51 \mathrm{a}$ & $10.2048 \pm 0.0659 \mathrm{a}$ \\
\hline & & $5^{\text {th }}$ & $6382.12 \pm 182.64 \mathrm{a}$ & $0.9254 \pm 0.0024 \mathrm{a}$ & $4053.87 \pm 66.01 \mathrm{~b}$ & $233.84 \pm 8.58 \mathrm{a}$ & $10.1088 \pm 0.1166 \mathrm{ab}$ \\
\hline & & $6^{\text {th }}$ & $5296.43 \pm 358.53 \mathrm{~b}$ & $0.9388 \pm 0.0045 \mathrm{~b}$ & $3331.77 \pm 247.87 \mathrm{c}$ & $202.86 \pm 10.93 \mathrm{~b}$ & $9.6289 \pm 0.2638 \mathrm{~b}$ \\
\hline \multirow{18}{*}{ Treatment } & \multirow{6}{*}{ A } & $1^{\mathrm{st}}$ & $7036.71 \pm 250.29 \mathrm{ab}$ & $0.9191 \pm 0.0030 \mathrm{ab}$ & $4238.83 \pm 187.42 \mathrm{ab}$ & $279.26 \pm 8.34 \mathrm{abc}$ & $10.2252 \pm 0.2024 \mathrm{ab}$ \\
\hline & & $2^{\text {nd }}$ & $7097.58 \pm 296.53 \mathrm{ab}$ & $0.9182 \pm 0.0035 \mathrm{ab}$ & $4438.20 \pm 180.44 \mathrm{ab}$ & $285.79 \pm 12.44 \mathrm{abc}$ & $10.6096 \pm 0.1222 \mathrm{ab}$ \\
\hline & & $3^{\text {rd }}$ & $6970.87 \pm 69.04 \mathrm{a}$ & $0.9202 \pm 0.0008 \mathrm{a}$ & $4332.83 \pm 18.36 \mathrm{a}$ & $281.57 \pm 2.71 \mathrm{a}$ & $10.4816 \pm 0.0813 \mathrm{ab}$ \\
\hline & & $4^{\text {th }}$ & $6779.91 \pm 171.66 \mathrm{ab}$ & $0.9226 \pm 0.0023 \mathrm{ab}$ & $4361.27 \pm 75.21 \mathrm{a}$ & $272.95 \pm 1.00 \mathrm{~b}$ & $10.5911 \pm 0.0405 \mathrm{a}$ \\
\hline & & $5^{\text {th }}$ & $6785.74 \pm 144.98 \mathrm{ab}$ & $0.9217 \pm 0.0016 \mathrm{ab}$ & $4288.40 \pm 62.93 \mathrm{a}$ & $273.31 \pm 6.35 \mathrm{abc}$ & $10.5010 \pm 0.0804 \mathrm{ab}$ \\
\hline & & $6^{\text {th }}$ & $6687.07 \pm 55.98 \mathrm{~b}$ & $0.9246 \pm 0.0004 \mathrm{~b}$ & $4141.67 \pm 11.93 \mathrm{~b}$ & $266.27 \pm 3.03 \mathrm{c}$ & $10.4958 \pm 0.0096 \mathrm{~b}$ \\
\hline & \multirow{6}{*}{ B } & $1^{\text {st }}$ & $6216.34 \pm 265.52 \mathrm{a}$ & $0.9253 \pm 0.0031 \mathrm{a}^{* *}$ & $3854.00 \pm 170.92 \mathrm{a}$ & $218.99 \pm 11.01 \mathrm{ab}$ & $10.1094 \pm 0.1668 \mathrm{a}$ \\
\hline & & $2^{\text {nd }}$ & $6172.83 \pm 380.08 \mathrm{a}^{*}$ & $0.9258 \pm 0.0044 \mathrm{a}^{*}$ & $3769.70 \pm 245.60 \mathrm{a}^{*}$ & $218.51 \pm 11.04 \mathrm{ab}$ & $10.0789 \pm 0.2209 \mathrm{a}^{* *}$ \\
\hline & & $3^{\text {rd }}$ & $5995.03 \pm 227.41 \mathrm{a}$ & $0.9289 \pm 0.0030 \mathrm{a}^{*}$ & $3693.07 \pm 93.42 \mathrm{a}^{*}$ & $215.77 \pm 1.35 \mathrm{a}$ & $10.0762 \pm 0.0440 \mathrm{a}$ \\
\hline & & $4^{\text {th }}$ & $5667.12 \pm 274.48 \mathrm{a}^{*}$ & $0.9331 \pm 0.0037 \mathrm{a}$ & $3536.70 \pm 173.22 \mathrm{a}^{* *}$ & $201.59 \pm 3.98 \mathrm{~b}^{* *}$ & $10.0396 \pm 0.0878 \mathrm{a}$ \\
\hline & & $5^{\text {th }}$ & $6116.57 \pm 332.40 \mathrm{a}$ & $0.9275 \pm 0.0043 \mathrm{a}^{*}$ & $3871.60 \pm 210.14 \mathrm{a}$ & $218.98 \pm 7.72 \mathrm{a}$ & $10.2730 \pm 0.1283 \mathrm{a}$ \\
\hline & & $6^{\text {th }}$ & $4476.10 \pm 142.60 \mathrm{~b}^{*}$ & $0.9474 \pm 0.0015 b^{* *}$ & $2831.57 \pm 173.20 \mathrm{~b}^{*}$ & $174.40 \pm 8.45 \mathrm{c}^{*}$ & $9.2518 \pm 0.3060 \mathrm{~b}$ \\
\hline & \multirow{6}{*}{$\mathrm{C}$} & $1^{\text {st }}$ & $6569.09 \pm 121.24 *$ & $0.9342 \pm 0.0012 *$ & $4075.93 \pm 124.77$ & $287.46 \pm 9.71 * *$ & $10.1284 \pm 0.1040$ \\
\hline & & $2^{\text {nd }}$ & $6019.10 \pm 504.64$ & $0.9407 \pm 0.0059$ & $3826.73 \pm 247.48$ & $268.80 \pm 20.43 *$ & $10.0742 \pm 0.0861$ \\
\hline & & $3^{\text {rd }}$ & $6508.00 \pm 278.96$ & $0.9346 \pm 0.0029 * *$ & $4039.37 \pm 158.86$ & $277.71 \pm 7.43 * *$ & $10.1280 \pm 0.0964$ \\
\hline & & $4^{\text {th }}$ & $6516.78 \pm 100.40$ & $0.9353 \pm 0.0015 *$ & $4106.73 \pm 134.61$ & $277.30 \pm 9.21 * *$ & $10.2449 \pm 0.0865$ \\
\hline & & $5^{\text {th }}$ & $6182.17 \pm 598.71$ & $0.9387 \pm 0.0060$ & $3852.07 \pm 379.64$ & $266.26 \pm 15.44$ & $9.9880 \pm 0.3112$ \\
\hline & & $6^{\text {th }}$ & $5964.58 \pm 540.95$ & $0.9414 \pm 0.0068$ & $3838.57 \pm 341.12$ & $275.25 \pm 18.38 * *$ & $10.1131 \pm 0.1931$ \\
\hline
\end{tabular}

the genus level, relative abundance of those identified at genus level was very low.

\section{Co-Occurrence Network Analysis}

Co-occurrence network analysis of the complexity of interactions between taxa detected in soils with and without straw return revealed some differences across regions. Higher co-occurrence network complexity was identified at location $\mathrm{C}$, relative to $\mathrm{B}$ and $\mathrm{A}$ (Fig. 7).
Among the taxa in these 3 networks, Proteobacteria and Acidobacteria showed their key positions in interaction.

\section{Relationships between Species Aabundance and Environmental Factors}

RDA results revealed strong correlation between species data and environmental factors, with speciesenvironment correlations on $1^{\text {st }}$ and $2^{\text {nd }}$ axes. Total $\mathrm{P}$ content was the dominant environmental variable 
a)

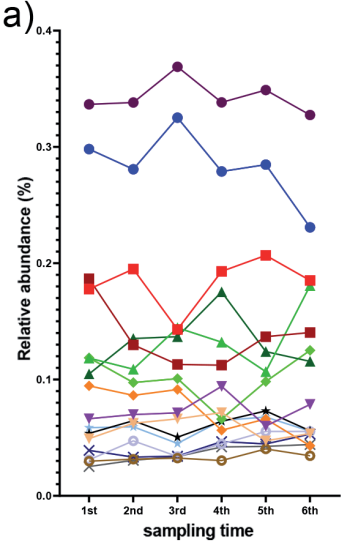

b)

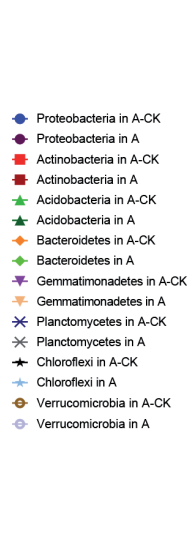

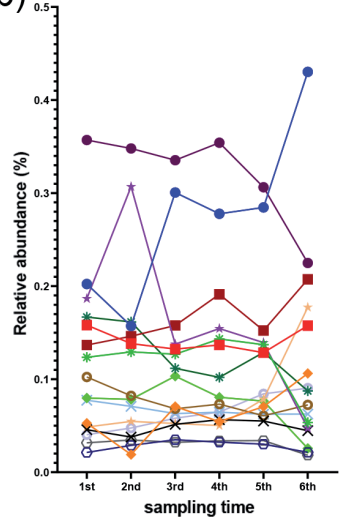

c)

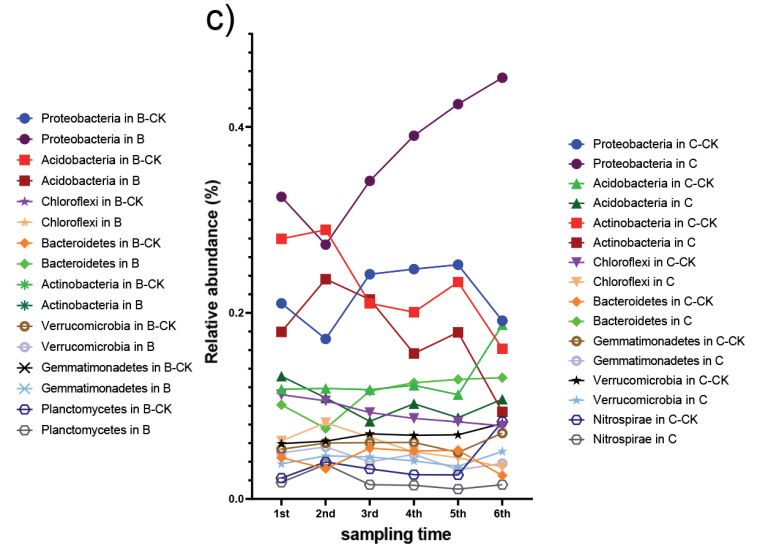

Fig. 6. Relative abundance of dominant bacteria. a), b), and c) notes soil samples in Songyuan, Dunhua and Jiutai, respectively. -CK notes soil samples without straw return treatment. All bacteria were counted at the phylum level.
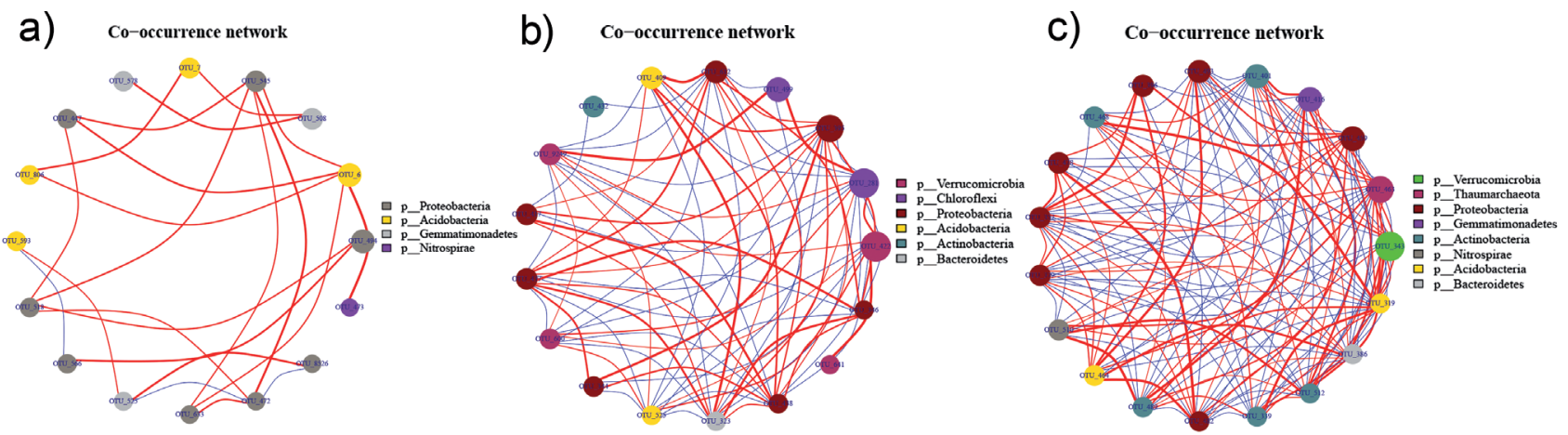

Fig. 7. Co-occurrence network analysis of bacterial OTUs in CK and short-term straw returning treatment samples. a), b), and c) notes the network's location (Songyuan, Dunhua, and Jiutai, respectively).

and mainly correlating with the relative abundance of Rhodanobacter, Burkholderia-Paraburkholderia, and Bryobacter_Bradyrhizobium. PH was the other dominant environmental variable correlating with the relative abundance of Acidbacter, RB41, Haliangium. Species like Acidothermus, Candidatus solibacter, H16, and Nitrospira negatively correlated with soil enzyme activity. Sphingomonas, Pedobacter, Gemmatimonas, and Pseudarthrobacter positively correlated with soil enzyme activity. However, the total $\mathrm{N}$ and soil $\mathrm{OM}$ content did not significantly correlate with bacterial abundance (Fig. 8).

\section{Discussion}

Crop straw is an important source of organic carbon in Chinese agro-ecosystems [24]. Returning crop straw to soil is a critical means of countering carbon loss due to mineralization in agricultural soils [25, 26]. Here, we find increased OM content after short-term straw returning at all experiment locations and sampling times, which is consistent with Chen's report [27]. It is likely that many nutrients and soluble $\mathrm{OM}$ in crop straw are released to soil, resulting in a virtuous circle with soil microorganisms [28]. Additionally, we found that the OM contents peaked at the $3^{\text {rd }}$ (Aug 15) or $4^{\text {th }}$ (Seq 9) time, and troughed at the $1^{\text {st }}$ or $6^{\text {th }}$ time, which may be due to temperature effects and effective accumulated temperature $[29,30]$. Higher temperature increased microbial activity and favored soil $\mathrm{OM}$ formation. In the experimental area, temperature initially rose before falling, peaking at the end of July. The accumulated temperature reached higher values in September. Since OM change at 3 locations was relatively consistent, we inferred that temperature and accumulated temperature jointly affected $\mathrm{OM}$ change. $\mathrm{OM}$ is known to be affected by a wide range of soil microorganisms. RDA results did not reveal significant positive correlation between soil OM content and soil microorganisms. Higher temperatures are expected to enhance soil bacteria activity, resulting in higher enzyme activity [31]. Enzyme activity peaked at the $2^{\text {nd }}$ or $3^{\text {rd }}$ sampling, likely due to higher temperature.

Short-term straw returning significantly lowered $\mathrm{pH}$ at all locations, although the 3 areas are far away from each other. This was consistent with past findings that $\mathrm{pH}$ value decreased (but not significantly) after 3 years of maize straw application [32]. The lower soil $\mathrm{pH}$ was an important factor for increased fungal 


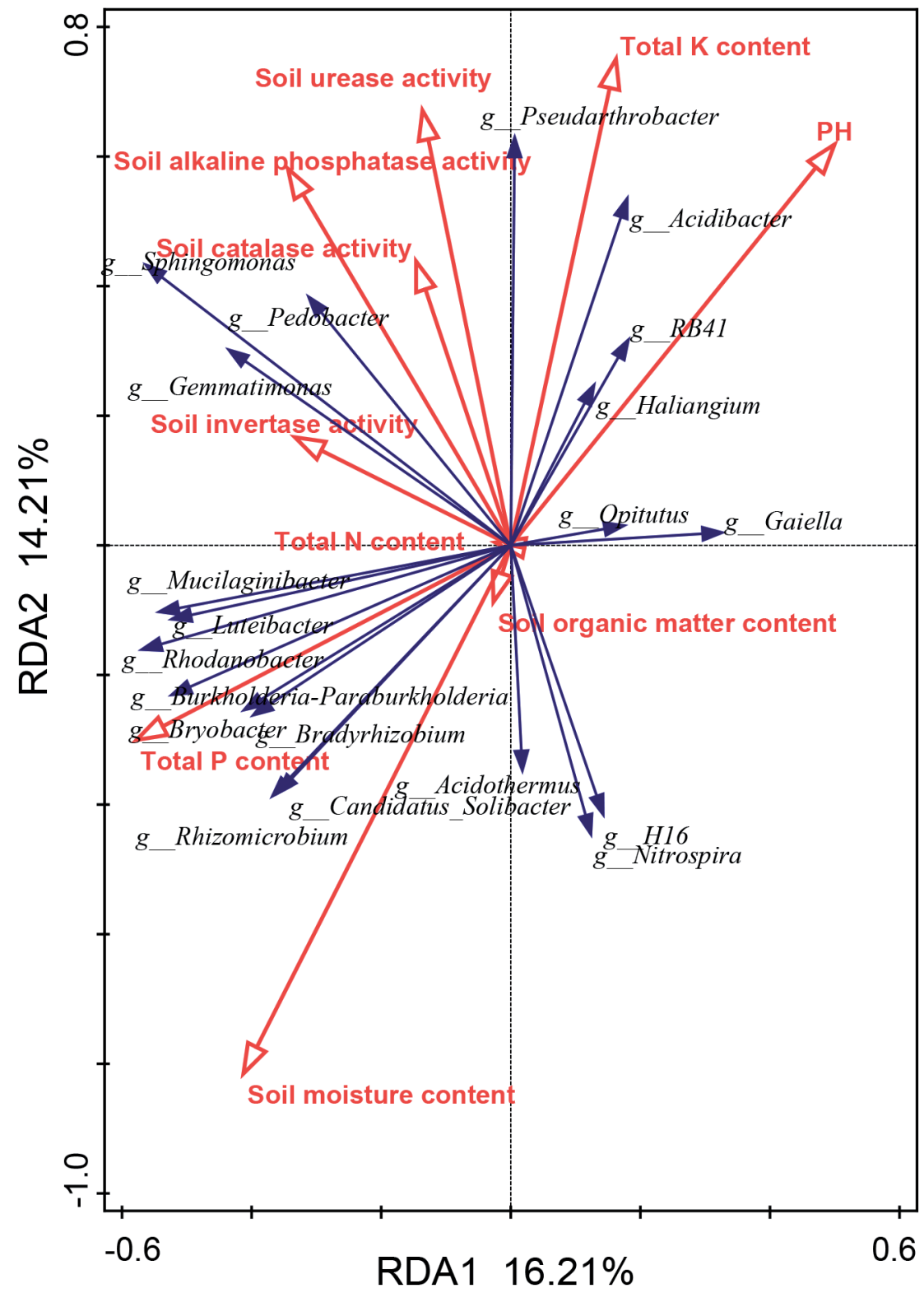

Fig. 8. Ordination diagram showing the results from redundancy analysis of species abundance and bacteria species abundance. The arrow length represents the strength of the correlation between the soil physical and chemical parameters and the bacteria. The longer the arrow length, the stronger the correlation. The perpendicular distance between the soil physical and chemical parameters and the bacteria axes in the plot reflects their correlations. The smaller the distance, the stronger the correlation.

abundance [33] as fungi prefer acidic environments [34]. Interestingly, $\mathrm{pH}$ positively correlated with Acidibacter, RB41, and Haliangium (Fig. 7), whose abundance may influence soil $\mathrm{pH}$ at different periods. SWC analysis revealed good water retention after soil treatment with short-term straw returning. Soil enzymes are produced and secreted by soil microorganisms, and are proximate agents of $\mathrm{OM}$ formation and decomposition [35].

Short-term straw returning also increased enzyme activity. The increased activity of enzyme secreted by soil microorganisms may promote soil OM decomposition, meeting carbon and nitrogen demand for microbial growth [36]. In general, OM contents and enzyme activities had a concerted trend that closely correlated to the amount and structure of soil microorganisms. Indicating that short-term straw returning enriches soil bacterial diversity. The $\alpha$-diversity of location B at $2^{\text {nd }}$ sampling had the lowest value in the control, but increased upon straw returning. Additionally, short-term straw returning could better maintain the stability of soil bacterial diversity, such as at location C. Different soil environments in the 3 regions might be the main cause of microbial diversity differences. Soil bacteria diversity might be affected by factors like temperature and $\mathrm{pH}$, especially for B and C control groups. However, straw returning significantly alleviated the influence of climate on soil 
microbial diversity. The alkalinity of area A soil and the faint acidity of area $\mathrm{B}$ and $\mathrm{C}$ soil might be one of the main reasons for changes in soil bacteria diversity. However, more evidence is needed to support this view. Short-term straw returning significantly increased the relative abundance of Proteobacteria and Bacteroidetes. Our data show that the bacterial phyla Proteobacteria and Bacteroidetes have similar dynamics upon straw application, which indicates that they are dominant in straw decomposition relative to other microbial fractions. A similar role has been reported for Proteobacteria [37]. Proteobacteria has been shown to consist of many classes that are sensitive to copiotrophic conditions [38]. Furthermore, the abundances of Delta-, Gamma- and Beta-Proteobacteria, were significantly improved by OM incorporation [39]. Past studies identified Bacteroidetes as the main microbial groups involved in breaking down the chemical components of rice straw, including cellulose, hemicellulose, and chitin [40]. Liu et al found that Bacteroidetes played an important role in degradation of the rice straw in paddy soils [41]. Thus, we speculated that Proteobacteria and Bacteroidetes are the important phyla during straw degradation, and that they promote soil OM accumulation and enhance soil fertility. On the contrary, the dominant phyla, Actinobacteria and Chloroflexi were reduced by straw returning, indicating that straw returning negatively affects their growth environment. Chloroflexi is another major microbial group that breaks down chemical components of crop straw [40]. Proteobacteria and Actinobacteria are copiotrophic taxa (taxa that thrive in conditions of elevated $\mathrm{C}$ and $\mathrm{N}$ and that exhibit relatively rapid growth rates) [42-44]. The decomposition process is conceptually separated by a rapid and a slower phase into the early and the late stage, respectively [45]. The organic components of plant residues can be easily degradable, such as long/ short chain fatty acids and less-degradable or more persistent to treatment fractions, including cellulose and lignin [46]. Straw input provides $\mathrm{C}$ for soil microbial growth, which promotes microbial growth [47]. We speculate that decreased Actinobacteria and Chloroflexi may be due to competition from dominant bacteria.

The enzyme activity associated with straw residues represents a key biological process that is closely related to nutrient absorption by microorganisms for their own metabolism [48]. In general, some watersoluble compounds and easily decomposed substances like sugar and starch, are important for high microbial activity, because they are $\mathrm{N}$-acetyl-glucosamine kinase and L-leucine aminopeptidase suitable substrates [49]. Here, enzyme activity gradually increased in the early stage. Additionally, Sphingomonas, Pedobacter, Gemmatimonas and Pseudarthrobacter, positively correlated with soil enzyme activity. Therefore, these bacteria may benefit the most from straw decomposition.

\section{Conclusion}

Short-term straw returning to the field can markedly improve soil quality, mainly reflected in the increasing of $\mathrm{OM}$ content and soil enzymes activity. Straw returning treatment might maintain soil stability and bacterial diversity better. The bacterial phyla Proteobacteria and Bacteroidetes, exhibited similar dynamics upon straw application, indicating that they are dominant over other microbial fractions during straw decomposition. Actinobacteria and Chloroflexi decreased after straw returning, indicating that straw returning negatively affects the growth environments of these bacteria. Decreased Actinobacteria and Chloroflexi may be due to competition from dominant bacteria. However, soil responses to straw returning differ by location, depending on basic soil parameters and climate.

\section{Core Ideas}

1. Short-term returning of straw can significantly improve soil quality.

2. The effect of short-term straw return is affected by basic soil conditions and climate.

3. Short-term straw returning has a significant regulatory effect on soil bacteria.

4. Proteobacteria and Bacteroidetes phyla may be dominant in straw decomposition.

\section{Abbreviations}

OM, organic matter;

OTUs, Operational taxonomic units;

PE, pair-end;

PCoA, Principal Co-ordinates Analysis;

qPCR, Quantitative PCR;

RDP, Ribosomal Database Project;

RDA, redundancy analysis;

SWC, Soil water content.

\section{Conflict of Interest}

The authors declare that they have no conflict of interest.

\section{Funding}

This research was supported by the National Key $\mathrm{R}$ \& D Program "Establishment of key technical models for improving yield and efficiency of spring maize" of China during the $13^{\text {th }}$ Five-Year Plan period (2017YFD0300607). 


\section{Supplementary Material}

a)

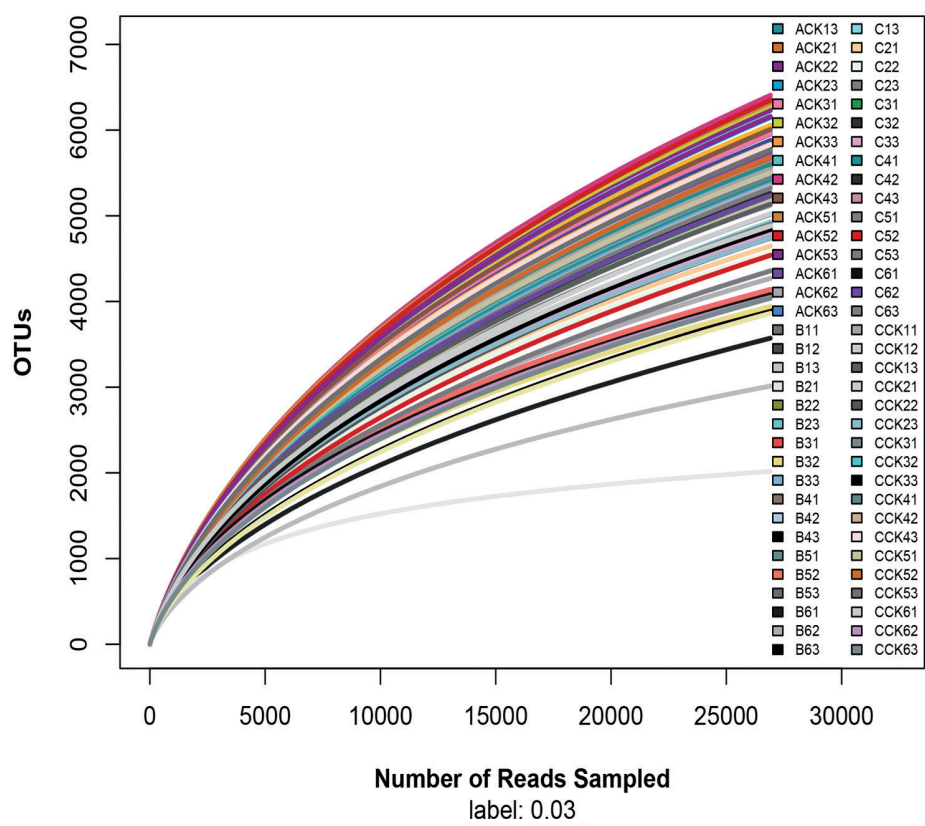

b)

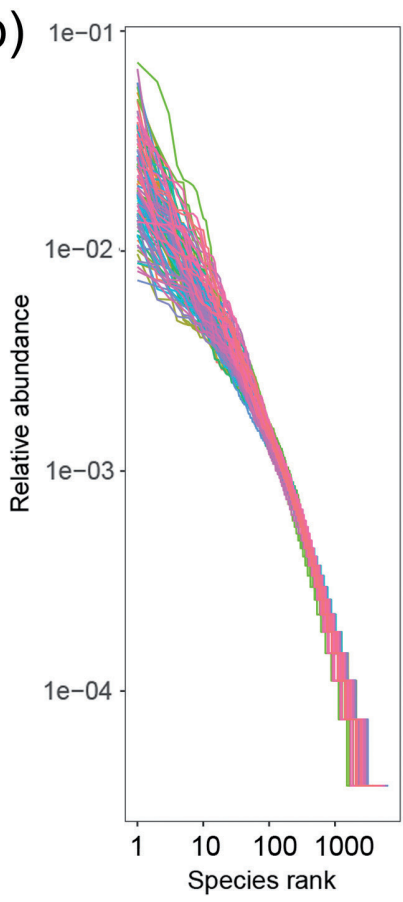

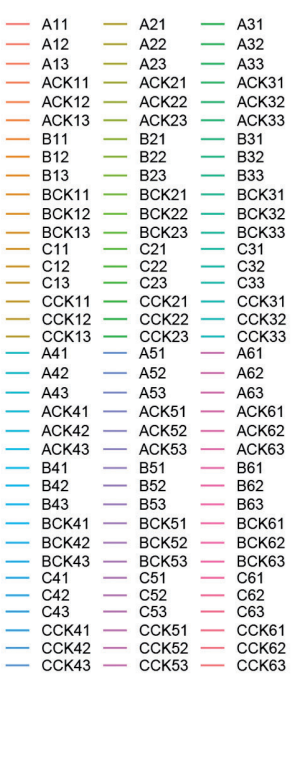

Fig. S1. Rarefaction curves and rank abundance curves of alpha diversity. a) Rarefaction curves plot, X-axis is number of sequencing reads randomly chosen from a certain sample to obtain OTUs. b) Rank abundance curves plot. X-axis shows abundance rank. Y-axis shows relative abundance. Curves for different samples are shown in different colors.

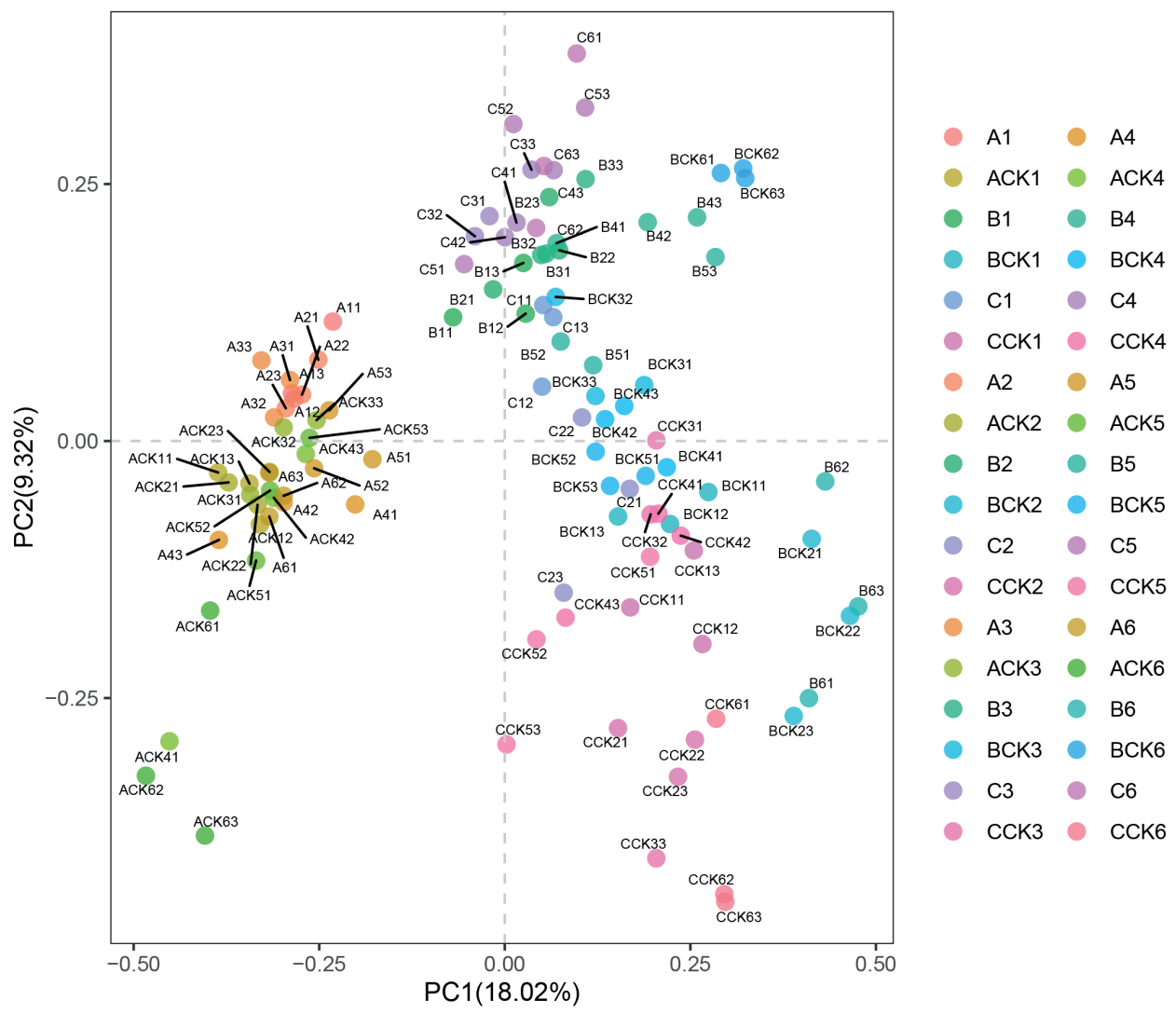

Fig. S2. Principle component analysis (PCA) for bacterial community 16S rRNA gene sequences. A, B, and C denote soil samples from Songyuan, Dunhua, and Jiutai, respectively. CK denotes soil samples without straw return. Different samples are shown in different colors. 
Table S1. Sequence data summary. A, B, and C denotes soil samples from Songyuan, Dunhua, and Jiutai, respectively. CK denotes soil samples without straw return.

\begin{tabular}{|c|c|c|c|}
\hline SampleID & Raw_Tags & Clean_Tags & OTUs \\
\hline A1_1 & 78955 & 76544 & 5455 \\
\hline A1_2 & 169550 & 164681 & 5877 \\
\hline A1_3 & 117986 & 114469 & 5668 \\
\hline ACK1_1 & 90273 & 87849 & 6328 \\
\hline ACK1_2 & 65232 & 63434 & 6162 \\
\hline ACK1_3 & 197948 & 192535 & 6008 \\
\hline B1_1 & 168652 & 164244 & 5580 \\
\hline B1_2 & 101581 & 98573 & 5384 \\
\hline B1_3 & 93920 & 90960 & 5138 \\
\hline BCK1_1 & 65012 & 63686 & 4876 \\
\hline BCK1_2 & 152772 & 149703 & 5150 \\
\hline BCK1_3 & 83521 & 81458 & 5469 \\
\hline $\mathrm{C} 1 \_1$ & 78079 & 76218 & 5371 \\
\hline C1_2 & 54844 & 53433 & 5353 \\
\hline C1_3 & 188046 & 183304 & 4930 \\
\hline CCK1_1 & 123663 & 121033 & 5546 \\
\hline CCK1_2 & 95934 & 94060 & 5025 \\
\hline CCK1_3 & 69875 & 68072 & 5136 \\
\hline A2_1 & 67515 & 65787 & 5604 \\
\hline A $2 \_2$ & 127991 & 124601 & 6056 \\
\hline A2 33 & 89746 & 87309 & 5953 \\
\hline ACK2_1 & 70980 & 69350 & 6372 \\
\hline ACK2_2 & 50391 & 49178 & 6234 \\
\hline ACK2_3 & 134162 & 130693 & 6148 \\
\hline B2_1 & 122028 & 119152 & 5590 \\
\hline B2 _2 & 83333 & 81260 & 5224 \\
\hline B2 _3 & 80404 & 78187 & 4860 \\
\hline BCK2_1 & 40451 & 39689 & 2016 \\
\hline BCK2_2 & 36655 & 35878 & 3013 \\
\hline BCK2_3 & 84648 & 83724 & 3862 \\
\hline $\mathrm{C} 2 \_1$ & 59685 & 57183 & 4647 \\
\hline C2 22 & 297317 & 290762 & 4566 \\
\hline $\mathrm{C} 2 \_3$ & 47668 & 46690 & 5320 \\
\hline CCK2_1 & 34963 & 34391 & 5480 \\
\hline CCK2_2 & 29350 & 28871 & 4744 \\
\hline CCK2_3 & 186142 & 183212 & 4744 \\
\hline A3_1 & 119051 & 115802 & 5803 \\
\hline A3 22 & 79472 & 77289 & 5663 \\
\hline A3_3 & 65839 & 64108 & 5691 \\
\hline
\end{tabular}

\begin{tabular}{|c|c|c|c|}
\hline SampleID & Raw_Tags & Clean_Tags & OTUs \\
\hline ACK3_1 & 46446 & 45293 & 5963 \\
\hline ACK3_2 & 103532 & 100150 & 6288 \\
\hline ACK3_3 & 112856 & 110062 & 5650 \\
\hline B3_1 & 77763 & 75736 & 5353 \\
\hline B3_2 & 63544 & 61697 & 5268 \\
\hline B3_3 & 70852 & 69271 & 4817 \\
\hline BCK3_1 & 129962 & 126259 & 5557 \\
\hline BCK3_2 & 88420 & 86014 & 5174 \\
\hline BCK3_3 & 75390 & 73218 & 5675 \\
\hline C3_1 & 53710 & 51692 & 5232 \\
\hline C3_2 & 157888 & 151982 & 5247 \\
\hline C3_3 & 124771 & 120020 & 4810 \\
\hline CCK3_1 & 75308 & 73035 & 5456 \\
\hline CCK3_2 & 70081 & 68052 & 5471 \\
\hline CCK3_3 & 168695 & 166938 & 4840 \\
\hline A4_1 & 43210 & 42201 & 6228 \\
\hline A4_2 & 30797 & 30071 & 5975 \\
\hline A4_3 & 97609 & 96026 & 5521 \\
\hline ACK4_1 & 328241 & 323468 & 5217 \\
\hline ACK4_2 & 43636 & 42341 & 6407 \\
\hline ACK4_3 & 34468 & 33470 & 6008 \\
\hline B4_1 & 29398 & 28640 & 5360 \\
\hline B4_2 & 182518 & 178390 & 4761 \\
\hline B4_3 & 56107 & 54700 & 4741 \\
\hline BCK4_1 & 91864 & 89355 & 5376 \\
\hline BCK4_2 & 52668 & 51010 & 5597 \\
\hline BCK4_3 & 58253 & 56733 & 5447 \\
\hline C4_1 & 35312 & 34032 & 5430 \\
\hline $\mathrm{C} 4 \_2$ & 107864 & 104135 & 5261 \\
\hline C4_3 & 86882 & 83887 & 4868 \\
\hline CCK4_1 & 57089 & 55459 & 5466 \\
\hline CCK4_2 & 47785 & 46502 & 5503 \\
\hline CCK4_3 & 68606 & 66965 & 5829 \\
\hline A5_1 & 165494 & 161328 & 5839 \\
\hline A5_2 & 91695 & 89056 & 5950 \\
\hline A5_3 & 89341 & 86893 & 5790 \\
\hline ACK5_1 & 64940 & 63392 & 6319 \\
\hline ACK5_2 & 162482 & 157884 & 6349 \\
\hline ACK5_3 & 119737 & 116077 & 6159 \\
\hline
\end{tabular}


Table S1. Continued.

\begin{tabular}{|c|c|c|c|}
\hline SampleID & Raw_Tags & Clean_Tags & OTUs \\
\hline B5_1 & 86066 & 83807 & 5835 \\
\hline B5_2 & 89705 & 87486 & 5609 \\
\hline B5_3 & 76864 & 74771 & 5018 \\
\hline BCK5_1 & 134055 & 130313 & 5400 \\
\hline BCK5_2 & 90210 & 87717 & 5683 \\
\hline BCK5_3 & 61842 & 60025 & 5259 \\
\hline C5_1 & 120113 & 115047 & 5681 \\
\hline C5_2 & 127740 & 122855 & 4539 \\
\hline C5_3 & 93715 & 90486 & 4357 \\
\hline CCK5_1 & 75321 & 73195 & 5501 \\
\hline CCK5_2 & 82367 & 80600 & 5687 \\
\hline CCK5_3 & 172346 & 168706 & 5770 \\
\hline A6_1 & 117008 & 114025 & 5652 \\
\hline A6_2 & 102372 & 99926 & 5495 \\
\hline A6_3 & 64499 & 62639 & 5755 \\
\hline
\end{tabular}

\section{References}

1. WEN D., LIANG W. Soil Fertility Quality and Agricultural Sustainable Development in the Black Soil Region of Northeast China. Environment, Development and Sustainability, 3 (1), 31, 2001.

2. LI BEI D.G., ZHAO PENGFEI, ZHOU DAN, ZHAO CHUANG, MI GUOHUA, ZHANG WEIFENG Effect of conservation practices on soil organic matter in medium or low fertility black soil. Journal of Agro-Environment Science, 39, 891, 2020.

3. MALHI S.S., NYBORG M., GODDARD T., PUURVEEN D. Long-term tillage, straw management and $\mathrm{N}$ fertilization effects on quantity and quality of organic $\mathrm{C}$ and $\mathrm{N}$ in a Black Chernozem soil. Nutrient Cycling in Agroecosystems, 90 (2), 227, 2011.

4. HANSEN V., MüLLER-STöVER D., MUNKHOLM L.J., PELTRE C., HAUGGAARD-NIELSEN H., JENSEN L.S. The effect of straw and wood gasification biochar on carbon sequestration, selected soil fertility indicators and functional groups in soil: An incubation study. Geoderma, 269, 99, 2016.

5. LIU E., YAN C., MEI X., HE W., BING S.H., DING L., LIU Q., LIU S., FAN T. Long-term effect of chemical fertilizer, straw, and manure on soil chemical and biological properties in northwest China. Geoderma, $\mathbf{1 5 8}$ (3), 173, 2010.

6. GONG W., YAN X., WANG J., HU T., GONG Y. Longterm manure and fertilizer effects on soil organic matter fractions and microbes under a wheat-maize cropping system in northern China. Geoderma, 149 (3), 318, 2009.

7. HE Y.T., ZHANG W.J., XU M.G., TONG X.G., SUN F.X., WANG J.Z., HUANG S.M., ZHU P., HE X.H. Long-term combined chemical and manure fertilizations increase soil organic carbon and total nitrogen in aggregate fractions at

\begin{tabular}{|c|c|c|c|}
\hline SampleID & Raw_Tags & Clean_Tags & OTUs \\
\hline ACK6_1 & 214836 & 210071 & 5733 \\
\hline ACK6_2 & 296939 & 294317 & 5123 \\
\hline ACK6_3 & 192603 & 191013 & 4766 \\
\hline B6_1 & 136316 & 135097 & 3572 \\
\hline B6_2 & 147636 & 144876 & 4270 \\
\hline B6_3 & 261378 & 257343 & 3901 \\
\hline BCK6_1 & 228666 & 222726 & 3944 \\
\hline BCK6_2 & 163401 & 159922 & 4132 \\
\hline BCK6_3 & 161480 & 157543 & 4141 \\
\hline C6_1 & 238724 & 231019 & 4079 \\
\hline C6_2 & 272008 & 263796 & 5235 \\
\hline C6_3 & 235502 & 228663 & 4906 \\
\hline CCK6_1 & 214183 & 209990 & 4881 \\
\hline CCK6_2 & 93941 & 93297 & 4047 \\
\hline CCK6_3 & 138349 & 137424 & 4038 \\
\hline
\end{tabular}

three typical cropland soils in China. Science of The Total Environment, 532, 635, 2015.

8. GUO S., WU J., COLEMAN K., ZHU H., LI Y., LIU W. Soil organic carbon dynamics in a dryland cereal cropping system of the Loess Plateau under long-term nitrogen fertilizer applications. Plant and Soil, 353 (1), 321, 2012.

9. YANG X., REN W., SUN B., ZHANG S. Effects of contrasting soil management regimes on total and labile soil organic carbon fractions in a loess soil in China. Geoderma, 177-178, 49, 2012.

10. JACOBY R., PEUKERT M., SUCCURRO A., KOPRIVOVA A., KOPRIVA S. The Role of Soil Microorganisms in Plant Mineral Nutrition-Current Knowledge and Future Directions. Frontiers in Plant Science, 8, 1617, 2017.

11. KIBOI M.N., NGETICH K.F., MUGENDI D.N., MURIUKI A., ADAMTEY N., FLIESSBACH A. Microbial biomass and acid phosphomonoesterase activity in soils of the Central Highlands of Kenya. Geoderma Regional, 15, e00193, 2018.

12. PATERSON E., SIM A., OSBORNE S.M., MURRAY P.J. Long-term exclusion of plant-inputs to soil reduces the functional capacity of microbial communities to mineralise recalcitrant root-derived carbon sources. Soil Biology and Biochemistry, 43 (9), 1873, 2011.

13. MARSCHNER P., UMAR S., BAUMANN K. The microbial community composition changes rapidly in the early stages of decomposition of wheat residue. Soil Biology and Biochemistry, 43 (2), 445, 2011.

14. KORANDA M., KAISER C., FUCHSLUEGER L., KITZLER B., SESSITSCH A., ZECHMEISTERBOLTENSTERN S., RICHTER A. Fungal and bacterial utilization of organic substrates depends on substrate complexity and $\mathrm{N}$ availability. FEMS Microbiology Ecology, 87 (1), 142, 2014. 
15. MAARASTAWI S.A., FRINDTE K., LINNARTZ M., KNIEF C. Crop rotation and straw application impact microbial communities in Italian and Philippine soils and the rhizosphere of Zea mays. Frontiers in Microbiology, 9, 1295, 2018

16. SUN L., XUN W., HUANG T., ZHANG G., GAO J., RAN W., LI D., SHEN Q., ZHANG R. Alteration of the soil bacterial community during parent material maturation driven by different fertilization treatments. Soil Biology and Biochemistry, 96, 207, 2016.

17. VOGEL C., BABIN D., PRONK G.J., HEISTER K., SMALLA K., KöGEL-KNABNER I. Establishment of macro-aggregates and organic matter turnover by microbial communities in long-term incubated artificial soils. Soil Biology and Biochemistry, 79, 57, 2014.

18. ZECHMEISTER-BOLTENSTERN S., KEIBLINGER K.M., MOOSHAMMER M., PEñUELAS J., RICHTER A., SARDANS J., WANEK W. The application of ecological stoichiometry to plant-microbial-soil organic matter transformations. Ecological Monographs, 85 (2), 133, 2015.

19. YANG L., LI T., LI F., LEMCOFF J., COHEN S. Fertilization regulates soil enzymatic activity and fertility dynamics in a cucumber field. Scientia Horticulturae, 116, 21, 2008.

20. GEISSELER D., HORWATH W. Regulation of Extracellular Protease Activity in Soil in Response to Different Sources and Concentrations of Nitrogen and Carbon. Soil Biology \& Biochemistry - SOIL BIOL BIOCHEM, 40, 3040, 2008.

21. BAO S., Soil and Agricultural Chemistry Analysis. 2000, Beijing: China Agriculture Press. 2000.

22. MIZRAHI-MAN O., DAVENPORT E.R., GILAD Y. Taxonomic Classification of Bacterial 16S rRNA Genes Using Short Sequencing Reads: Evaluation of Effective Study Designs. PLOS ONE, 8 (1), e53608, 2013.

23. YUAN Z.-Q., YU K.-L., WANG B.-X., ZHANG W.-Y., ZHANG X.-L., SIDDIQUE K.H.M., STEFANOVA K., TURNER N.C., LI F.-M. Cutting improves the productivity of lucerne-rich stands used in the revegetation of degraded arable land in a semi-arid environment. Scientific reports, 5, 12130, 2015

24. LIU C., LU M., CUI J., LI B., FANG C. Effects of straw carbon input on carbon dynamics in agricultural soils: a meta-analysis. Global Change Biology, 20 (5), 1366, 2014.

25. LI F., CAO X., ZHAO L., YANG F., WANG J., WANG S. Short-term effects of raw rice straw and its derived biochar on greenhouse gas emission in five typical soils in China. Soil Science and Plant Nutrition, 59 (5), 800, 2013.

26. CHEN L., ZHANG J., ZHAO B., YAN P., ZHOU G., XIN $X$. Effects of straw amendment and moisture on microbial communities in Chinese fluvo-aquic soil. Journal of Soils and Sediments, 14 (11), 1829, 2014.

27. CHEN Z., WANG H., LIU X., ZHAO X., LU D., ZHOU J., LI C. Changes in soil microbial community and organic carbon fractions under short-term straw return in a ricewheat cropping system. Soil and Tillage Research, 165, 121, 2017.

28. WANG W., LAI D.Y.F., WANG C., PAN T., ZENG C. Effects of rice straw incorporation on active soil organic carbon pools in a subtropical paddy field. Soil and Tillage Research, 152, 8, 2015.

29. SIERRA C.A., TRUMBORE S.E., DAVIDSON E.A., VICCA S., JANSSENS I. Sensitivity of decomposition rates of soil organic matter with respect to simultaneous changes in temperature and moisture. Journal of Advances in Modeling Earth Systems, 7 (1), 335, 2015.

30. MOINET G.Y.K., HUNT J.E., KIRSCHBAUM M.U.F., MORCOM C.P., MIDWOOD A.J., MILLARD P. The temperature sensitivity of soil organic matter decomposition is constrained by microbial access to substrates. Soil Biology and Biochemistry, 116, 333, 2018.

31. ZHANG X., ZHANG B., MIAO R., ZHOU J., YE L., JIA D., PENG W., YAN L., ZHANG X., TAN W., LI X. Influence of Temperature on the Bacterial Community in Substrate and Extracellular Enzyme Activity of Auricularia cornea. Mycobiology, 46 (3), 224, 2018.

32. WANG X., JIA Z., LIANG L., ZHAO Y., YANG B., DING R., WANG J., NIE J. Changes in soil characteristics and maize yield under straw returning system in dryland farming. Field Crops Research, 218, 11, 2018.

33. HE S.-C.Z.C.-Y.C.K.-J.L.S.-J.Q.W.Z.P. Effects of long-term straw return on soil fertility,nitrogen pool fractions and crop yields on a fluvo-aquic soil in North China. Journal of Plant Nutrition and Fertilizer, 06, 1441, 2014.

34. ROUSK J., BROOKES P.C., BÅ̊TH E. Fungal and bacterial growth responses to $\mathrm{N}$ fertilization and $\mathrm{pH}$ in the 150-year \&\#8216;Park Grass\&\#8217; UK grassland experiment. FEMS Microbiology Ecology, 76 (1), 89, 2011.

35. BURNS R.G., DEFOREST J.L., MARXSEN J., SINSABAUGH R.L., STROMBERGER M.E., WALLENSTEIN M.D., WEINTRAUB M.N., ZOPPINI A. Soil enzymes in a changing environment: Current knowledge and future directions. Soil Biology and Biochemistry, 58, 216, 2013.

36. LUO L., MENG H., GU J.-D. Microbial extracellular enzymes in biogeochemical cycling of ecosystems. Journal of Environmental Management, 197, 539, 2017.

37. ZHAO S., SHAO-JUN Q., XU X., CIAMPITTI I., ZHANG S., HE P. Change in straw decomposition rate and soil microbial community composition after straw addition in different long-term fertilization soils. Applied Soil Ecology, 2019.

38. WANG J., MA Y., DI L., QIAN X., WANG G. Straw Incorporation with Nitrogen Amendment Shapes Bacterial Community Structure in an Iron-Rich Paddy Soil by Altering Nitrogen Reserves. Microorganisms, 9 (5), 988, 2021.

39. BEI S., ZHANG Y., LI T., CHRISTIE P., LI X., ZHANG J. Response of the soil microbial community to different fertilizer inputs in a wheat-maize rotation on a calcareous soil. Agriculture, Ecosystems and Environment, 260, 58, 2018.

40. W W.C.L. Microbial community dynamics during the early stages of plant polymer breakdown in paddy soil. Environmental Microbiology, 18, 2825, 2015.

41. LIU Y.-R., DONG J.-X., HAN L.-L., ZHENG Y.-M., HE J.-Z. Influence of rice straw amendment on mercury methylation and nitrification in paddy soils. Environmental Pollution, 209, 53, 2016.

42. FIERER N., LAUBER C.L., RAMIREZ K.S., ZANEVELD J., BRADFORD M.A., KNIGHT R. Comparative metagenomic, phylogenetic and physiological analyses of soil microbial communities across nitrogen gradients. The ISME Journal, 6 (5), 1007, 2012.

43. DAI Z., SU W., CHEN H., BARBERáN A., ZHAO H., YU M., YU L., BROOKES P.C., SCHADT C.W., CHANG S.X. Long-term nitrogen fertilization decreases bacterial diversity and favors the growth of Actinobacteria and Proteobacteria in agro-ecosystems across the globe. Global change biology, 24 (8), 3452, 2018. 
44. WANG Y., JI H., GAO C. Differential responses of soil bacterial taxa to long-term $\mathrm{P}, \mathrm{N}$, and organic manure application. Journal of soils and sediments, 16 (3), 1046, 2016.

45. SU Y., HE Z., YANG Y., JIA S., YU M., CHEN X., SHEN A. Linking soil microbial community dynamics to straw-carbon distribution in soil organic carbon. Scientific reports, $\mathbf{1 0}(1), 1, \mathbf{2 0 2 0}$.

46. ZHAN Y., LIU W., BAO Y., ZHANG J., PETROPOULOS E., LI Z., LIN X., FENG Y. Fertilization shapes a wellorganized community of bacterial decomposers for accelerated paddy straw degradation. Scientific reports, 8 (1), 7981, 2018.

47. BRADFORD M.A., KEISER A.D., DAVIES C.A., MERSMANN C.A., STRICKLAND M.S. Empirical evidence that soil carbon formation from plant inputs is positively related to microbial growth. Biogeochemistry, 113 (1), 271, 2013.

48. MCBRIDE S.G., STRICKLAND M.S. Quorum sensing modulates microbial efficiency by regulating bacterial investment in nutrient acquisition enzymes. Soil Biology and Biochemistry, 136, 107514, 2019.

49. MCMAHON S.K., WILLIAMS M.A., BOTTOMLEY P.J., MYROLD D.D. Dynamics of Microbial Communities during Decomposition of Carbon-13 Labeled Ryegrass Fractions in Soil. Soil Science Society of America Journal, 69 (4), 1238, 2005. 
